\title{
Synthesis of artificial lymphoid tissue with immunological function.
}

AUTHOR(S):

Kobayashi, Yuka; Watanabe, Takeshi

\section{CITATION:}

Kobayashi, Yuka ...[et al]. Synthesis of artificial lymphoid tissue with immunological function.. Trends in immunology 2010, 31(11): 422-428

\section{ISSUE DATE:}

2010-11

URL:

http://hdl.handle.net/2433/131820

\section{RIGHT:}

(C) 2010 Elsevier Ltd; この論文は出版社版でありません。引用の際には 出版社版をご確認ご利用ください。; This is not the published version. Please cite only the published version. 
Review

Synthesis of artificial lymphoid tissues with immunological function

Yuka Kobayashi and Takeshi Watanabe

Graduate School of Medicine, Kyoto University, Yoshida-konoe machi, Sakyo-ku, Kyoto 606-8501 JAPAN

Corresponding Author: Dr Takeshi Watanabe, Graduate School of Medicine, Kyoto University. Yoshida-konoe machi, Sakyo-ku, Kyoto 606-8501 JAPAN

Email:wtakeshi@ak.med.kyoto-u.ac.jp 


\section{Abstract:}

The ability to generate functional artificial lymphoid tissue to induce specific immunity at ectopic sites would offer a potential breakthrough for treatment of diseases such as cancer and severe infection using immunotherapy. Artificial lymphoid tissue would also offer an informative tool to further study lymphoid tissue development and function in vivo. Here we review the process of secondary and tertiary lymphoid organization - for which an understanding will be essential for artificial lymphoid tissue synthesis. Using this knowledge, we consider the combination of cell types, soluble factors and scaffold properties that will enable proper accumulation and organization of lymphocytes into tissue grafts. Recent successes with trials for the in vivo induction of artificial lymphoid tissue are also considered. 


\section{Lymphoid tissue formation and induction of immunity}

The adaptive immune system responds to antigens to produce various outcomes such as tolerance, antigen-specific immunity and immunological memory. These immune responses are, in most cases, supported by secondary lymphoid organs (SLO), such as lymph nodes (LN), Peyer's patches (PP) and the spleen, as well as tertiary lymphoid organs (TLO). In contrast to SLO, which develop during embryogenesis ${ }^{1,2}$, TLO form in the postnatal period in response to antigenic stimuli ${ }^{3}$. Although TLO development is initiated by antigenic stimuli, the process partially recapitulates development of SLO during embryogenesis. For example, both SLO and TLO structures include segregated T and B cell accumulations, CD11c+ dendritic cell (DC) clusters, follicular dendritic cell (FDCs) networks, and high endothelial venules (HEV) ${ }^{3,4}$. TLO formation occurs at ectopic sites and often accompanies the onset of acute and chronic inflammation, infection, autoimmune diseases and tumorogenesis, to provide a highly localized immune response against microbial-derived or autoanitgens. Examples of TLO include lymphoid tissue formation in the synovial membrane of rheumatoid arthritis ${ }^{5-7}$, aorta adventitia of atherosclerosis ${ }^{8,9}$ and salivary gland of Sjögren syndrome ${ }^{10,11}$. TLOs have also been observed during organ transplant rejection, which raises the possibility that they contribute to T cell-dependent allograft rejection by serving as a site for local adaptive immune responses ${ }^{12}$. To address this question, RIP-LT $\alpha$, which offer a model for skin transplant were used. These mice express Lta (lymphotoxin $\alpha$ ) under the control of the rat insulin promoter (RIP) and develop TLOs in the pancreatic islets, kidney and skin. RIP-LT $\alpha$ mouse skin grafts (containing TLO) were transplanted into allogeneic splenectomized aly/aly host mice, which lack all SLO. The skin allograft with TLO containing host-derived lymphocytes was rejected. Thus, the TLOs support the 
activation of naïve $T$ cells to effector and memory $T$ cells. The findings provide rationale for developing strategies to artificially synthesize lymphoid tissues at ectopic local sites, where a strong and specific immune response is desired. To synthesize functional artificial lymphoid tissues (aLT) at ectopic sites in vivo or in vitro, the regulated processes of SLO and TLO formation must be precisely understood. It will also be important to make use of advances in tissue engineering, with respect to both new technologies and biomaterials. Here, we provide an overview of SLO and TLO development, and then discuss trials to date for the in vivo induction of aLT.

\section{Lymphoid tissue-inducer (LTi) cells and lymphoid organ formation}

The physiological processes that drive SLO development have been well studied ${ }^{1,2,13,14}$. During embryogenesis, lymphoid tissue formation is dependent on the interaction between hematopoietic CD45+ CD3- CD4+ LT $\alpha 1 \beta 2+$ LTi cells and VCAM-1+ ICAM-1+ LT $\beta R+$ stromal lymphoid tissue organizer (LTo) cells ${ }^{1,13,15}$. LTi-like cells also contribute to lymphoid organogenesis in the adult ${ }^{16}$. LTi cells express a variety of factors important for organogenesis including the surface molecules IL-7R $\alpha$, common gamma chain, CXCR5, RANKL (TRANCE) and RANK, and transcriptional factors such as RORC and Id $2^{16-22}$. Since both the mature embryonic LTi cells and adult LTi cells are recruited and maintained at the lymphoid tissue anlagen (during embroyogenesis) and at sites of chronic inflammation or infection (in the adult) ${ }^{18}$, they require the capacity to migrate through the vasculature. Indeed, whole embryonic spleens implanted under the renal subcapsular space of adult mice induce lymphoid tissue formation through accumulation of host-derived T cells, B cells and DCs. Host LTi cells are also recruited, which leads to stimulation of the grafted LTo cells and the production of CCL21 which 
attracts naïve T cells and DCs ${ }^{23}$. These findings suggest that the circulating adult LTi cells migrate into the grafts and interact with embryonic stromal cells to organize splenic white pulp formation ${ }^{23}$. In human fetal tissues, LTi cells have been identified as lineage- RORC+ IL-7R $\alpha+$ hematopietic cells. They are able to stimulate the expression of VCAM-1 and ICAM-1 on human mesenchymal stem cells in vitro, through expression of LT $\alpha 1 \beta 2$, which triggers LT $\beta R$ signaling in LTo cells ${ }^{24}$.

\section{LTo in SLO and TLO}

In contrast to LTi cells, the VCAM-1+ ICAM-1+ LT $\beta$ R+ LTo stromal cells are adherent cells that are non-hematopoietic and can be categorized according to heterogeneous functions ${ }^{25}$. In SLO and TLO, two important stromal cells play a specialized role in lymphoid tissue formation: the FDC in B cell follicles and the fibroblastic reticular cells (FRC) in the T cell area. Their precise immunological contributions have been detailed in a recent review ${ }^{25}$. In the T cell area, FRC networks, and their conduit system, form three-dimensional structures that provide tracks for T cell migration, which is regulated by CCR7. FRCs are the source of survival factors such as CCL19, IL-6 and IL-7, as well as mediators such as $\operatorname{VEGF}^{26}$ and retinoic acid (RA) that is produced by the enzyme RALDH2 ${ }^{27,28}$. RA leads to homing of activated T cells through activation of DCs ${ }^{29}$. VEGF induces proliferation of endothelial cells and maintains blood vessels ${ }^{25}$. The conduit system is sheathed and interconnected by FRC and is also linked to lymphatic vessels with fluid flow at draining lymph nodes (LN). Using three dimensional LN T cell areas that were constructed in vitro using FRC clones cultured together with type I collagen and matrigel, it was shown that fluid flow in the in vitro structures was required for CCL21 secretion from FRC, which enhanced the organization of FRC networks ${ }^{30}$. In vivo 
analysis by the same authors also indicated that blocking lymph flow in peripheral LN decreased CCL21 and CCL19 gene expression in FRC. These data suggest that under conditions of increased lymph flow, for example during inflammation, FRC networks increase in complexity, which could activate immune cell trafficking, lymph sampling and induction of immune responses ${ }^{30}$. In inflammation foci, it has been shown that local resident fibroblasts ${ }^{31}$ and aortic smooth muscle cells ${ }^{8,9}$ give rise to LTo-like stromal cells, which express chemokines such as CXCL13, CCl19 and CCL21 and recapitulate SLO formation after triggering of the LT $\beta R$ signaling pathway. Very recently, it was reported that lymph node FRC ectopically express and directly present peripheral tissue antigen (PTA) to naïve $T$ cells under steady-state as well as inflammatory conditions ${ }^{32}$. Another report also documented that lymph node-resident lymphatic endothelial cells directly present PTA to T cells and mediate peripheral tolerance in an Aire-independent manner ${ }^{33}$. These reports suggest that diverse stromal cell types exist that constitutively express PTA, and that lymph node stromal cells are involved in the maintenance of tolerance to self-antigens in the periphery. Thus, for the purpose of generating aLT with different immunological functions, including regulatory activity, it will be crucial to clarify the stromal cell heterogeneity in lymphoid organs.

\section{Maintaining a microenvironment for lymphoid tissues formation}

The initiation of lymphoid organ formation is triggered by the interaction of lymphotoxin $\alpha 1 \beta 2$ (LT $\alpha 1 \beta 2$ ) expressed on LTi cells with lymphotoxin- $\beta$ receptor (LT $\beta R$ ) on the LTo cells at the lymphoid anlagen, which leads to activation of the NF- $\kappa B$ signaling pathway. LT $\beta R$ ligation activates expression of the adhesion molecules VCAM-1, ICAM-1 and MAdCAM-1, as well as lymphoid chemokines such as CCL19, 
CCL21 and CXCL13 ${ }^{34,35}$. LT $\beta$ R signaling events also promotes LTo cell expression of RANKL and IL-7, and binding of these ligands to their respective receptors on the newly recruited immature LTi cells acts to further induce LT $\alpha 1 \beta 2$. This provides a positive feedback loop for lymphoid organ development at anlagen. Furthermore, the production of VEGF-C is induced in LTo cells, which contributes to lymphangiogenesis at this developmental stage ${ }^{35}$, as shown in Figure-1A. LT $\beta R$ associated signaling molecules also include TRAF2, TRAF3, the classical IKB kinase complex containing IKK $\alpha$, IKK $\beta$, IKK $\gamma$, and ELKs and NF-KB-inducing kinase. Two recent studies suggest that TRAF3 is a dual-mode regulator acting downstream of LT $\beta R$ to operate both canonical and non-canonical NF- $\mathrm{KB}$ signaling pathways ${ }^{36}$, and is critical for B cell homeostasis in SLO 37. Thus, LT $\beta R$ signaling broadly contributes not only to lymphoid organ development but also to the maintenance of proper microenvironments wherein hematopoietic cells can home and interact. In influenza virus-infected mouse respiratory tracts, ectopic lymphoid tissues known as inducible bronchus-associated lymphoid tissues (iBALT) form through accumulation of lymphocytes and dendritic cells. CD11c+ DC depletion from the lung leads to the gradual disappearance of B220+ cells from the iBALT structure and a decrease of peanut agglutinin (PNA)-positive germinal center B cells, followed by diminution in the level of local immunoglobulin class switching. This suggests that CD11C+ DCs support maintenance of iBALT structure and function ${ }^{38}$, indicating that CD11c+ DCs are involved in TLO organogenesis in addition to LTo stromal cells.

For proper lymphoid organogenesis, spatiotemporal regulation of the cells and molecules that localize and function in the lymphoid anlagen is required. Recently, it 
has been reported that heparan sulfate proteoglycans (HSPGs) play an important role in lymphoid development ${ }^{39}$. HSPGs are molecules that contribute to several regulatory mechanisms during tissue morphogenesis by specifically binding soluble factors. In this regard, a number of lymphoid chemokines contain HSPGs binding motifs ${ }^{40,41}$. Heparan sulfate (HS) glucuronyl C5-epimerase (Glce) mutant mice, which lack proper HS modifications, suffer from splenic hypoplasia, peripheral LN deletion, blood vessel mis-location and excess branching around peripheral $\mathrm{LNs}^{39}$. These results indicate that SLO development requires a tightly organized process that combines the signaling pathways required for accumulation of hematopoietic cells, together with suitable mechanisms for retention of both lymphoid cells and bioactive molecules within the developing lymphoid organ. It will be important to take these findings into account when generating synthetic immune tissues.

\section{Contributions of chemokines to lymphoid tissue generation}

Both SLO and TLO possess organized structures, which include T and B cell areas, DCs, FDC networks and vascular networks. To initiate SLO and TLO development and to sustain their function, lymphoid chemokines play important roles in the migration and proper positioning of these lymphoid tissue-associated cells [Figure 1B]. In mice, CCL19 and CCL21 expressed by FRC, and DCs attract and retain T cells within the appropriate area of the lymphoid tissues. Mice lacking both of CCL19 and CCL21, or mice lacking their receptor CCR7, retain LNs and PPs but exhibit defects in T cell trafficking to these lymphoid tissues, and in nasopharynx-associated lymphoid tissue maturation ${ }^{42,43}$. By performing immunohistochemical analysis of human SLO and TLO in inflammatory lesions, it was demonstrated that CCL19 and CCL21 are both expressed by the smooth 
muscle actin-positive stromal cells that are found in close proximity to HEVs, and CCL21 is also expressed by mature DCs and lymphatic vessels ${ }^{44}$. CXCL12, the ligand for CXCR4, also contributes to T cell trafficking into LNs and PPs in collaboration with CCR7 ligands 43. Another important chemokine is CXCL13, which is expressed by stromal cells including FDCs. CXCL13 initiates lymphoid organ formation ${ }^{45}$, entrance of B cells into follicles, and increased expression of LT $\alpha 1 \beta 2$ on B cells. CXCL13 further facilitates CXCL13 expression in B cells, establishing a positive feedback loop for B cell follicle homeostasis ${ }^{46}$. Mice deficient for both CXCL13 and IL-7R $\alpha$ lack most LNs, but CXCL13 is sufficient to recruit LTi cells to ectopic sites in vivo ${ }^{47}$. These results, along with the IL-7R $\alpha$-mediated LT $\alpha 1 \beta 2$ up-regulation on LTi cells, suggest that both CXCL13 and IL-7R $\alpha$ contribute to accumulation and activation of LTi cells respectively during the initial LT $\beta R$ signaling-dependent stage in LN development ${ }^{47}$. It has been shown, however, that in E12.5-14.5 LN anlagen, CXCL13 expression in LTo cells is induced by retinoic acid RA in a LT $\beta R$ signaling-independent manner. A possible source of RA appears to be cells of the nervous system around the LN anlagen ${ }^{45}$. In the SLO and TLO microenvironment, B cells circulate throughout B cell follicles to survey antigens. Once B cells engage antigens, the antigen-specific B cells increase CCR7 and CXCR5 expression, leading to their migration to the boundary between the $B$ cell follicle and $T$ cell zone, where B cells interact with antigen-specific $\mathrm{T}$ cells. Thus, controlled positioning of $B$ cells in follicles is regulated by the finely-tuned responsiveness of $B$ cells to chemokines such as CXCL13, CCL19 and CCL21 ${ }^{48}$. TLO formation was observed by ectopic expression of various chemokines such as CXCL12, CXCL13, CCL19 and CCL21 in transgenic mice ${ }^{49-51}$. Overexpression of each chemokine alone induced the complete formation of TLO. Ectopic expression of cytokines such as LT $\alpha \beta$ or IL-7 also formed TLO 
52,53. Thus, certain chemokines and cytokines might be important and useful for generating artificial lymphoid tissues.

\section{Scaffolds for artificial lymphoid tissues}

Tissue engineering has received much attention in the field of regenerative medicine and is undergoing continuous innovation. The characteristics of available biomaterials play an important role in tissue engineering strategies, and have allowed for the construction of a variety of tissues including blood vessels ${ }^{54,55}$, bone ${ }^{56,57}$, spinal cord ${ }^{58}$ and peripheral nervous system ${ }^{58,59}$. As a matter of course, this is now being applied to lymphoid tissues. Experimentally, to maintain the proper microenvironments at local sites, it will be necessary to attempt transplantation using scaffolds that: 1) contain the appropriate cell populations; 2) contribute to generation of lymphoid organs; 3 ) produce soluble factors - such as lymphoid chemokines and cytokines - that are expressed by stromal cells. A number of synthetic biomaterials are now able to duplicate the three dimensional microenvironments that are provided by natural extracellular matrices, such as fibrillar or non-fibrillar collagen, proteoglycans and matricellular proteins ${ }^{60,61}$. It has been demonstrated that structurally-engineered macroporous scaffolds, which combine poly ethylene glycal hydrogels with collagen, support T cell and DCs migration ${ }^{62}$. Further advances are being made in this area ${ }^{60,61}$. The first in vivo synthesis of artificial lymphoid tissues was achieved using a porous biocompatible collagen matrix, prepared from bovine Achilles tendon (referred to as a collagen sponge) ${ }^{63}$. This matrix has a non-homogeneous pore size ranging from $50-300 \mu \mathrm{m}$. A difficulty in tissue engineering is the achieving the ability to properly modulate or mimick, dynamic tissue microenvironments, for example to achieve 
appropriate cell recruitment or correct concentration gradients of soluble factors. As a first step toward this goal, biomaterials were recently described that are able to release several soluble factors, not only uniformly, but also gradually with temporal differences 64. In this study, two growth factors were incorporated together into the same scaffolds by mixing PDGF-encapsulated polymer, lyophilized VEGF and non-treated polymer particles, so that the two growth factors were localized within distinct compartments. As a result, the scaffold could release the two factors with distinct kinetics and, after transplantation into subcutaneous tissue of Lewis rats, more mature vascular networks were structured than when using the scaffold which contained only a single growth factor ${ }^{64}$. Two other recent studies have also reported that sustained ${ }^{65}$ or spatiotemporal ${ }^{66}$ delivery of growth factors can lead to formation of a mature vascular system. Thus, in order to efficiently generate artificial organized tissues, the scaffold microenvironment should be carefully constructed to be as close as possible to natural state.

\section{Generating artificial lymphoid tissue in vivo}

Generating functional artificial lymphoid tissues might be an important and beneficial method for making advances in immunotherapy, as well as for investigating the physiological functions of lymphoid tissues in vivo. For example, in the tumor microenvironment it is known that $\mathrm{T}$ cell immune responses are induced inside the tumor mass ${ }^{67}$. This, however, is not sufficient for tumor destruction due to immunosuppressive mechanisms. This immunosuppression arises from an imbalance between activating and inhibitory signals that regulate immune cell function, as well as overproduction of immunosuppressive cytokines such as TGF $\beta$ by the tumor mass ${ }^{68,69}$. 
Transplantation of aLT into the renal subcapsular space ${ }^{63,70}$ or close to the pathogenic site might help supplement anti-tumor immune responses. Another example where aLT might be beneficial to boost immune activity would be to counteract the atrophy of primary lymphoid organs and SLO which occurs during aging and which leads to immunosenescence ${ }^{71,72}$.

So far, tissue engineering has been most widely studied in a rodent model using splenic tissue. Several reports have shown that not only embryonic and newborn splenic transplantation ${ }^{23,73}$, but also tissue-engineered spleen (TES) generated from splenic multicellular components loaded on a biodegradable polymer scaffold ${ }^{74}$, induce partial or sufficient immune responses to infection. These studies provide valuable information concerning the generation of artificial lymphoid tissues. However, this approach lacks versatility in a clinical setting where the use of biological material might not be feasible if tissue supply is limited. Optimized combinations of stromal or hematopoietic cells and soluble factors such as chemokines or cytokines captured on scaffolds will be required to achieve solid results with general versatility. To date, few studies have managed to combine structural formation of aLT with immune responsiveness. However, we transplantated a collagen sponge that contained the thymus-derived cell line TEL-2 that overexpressed LT $\alpha$, stromal cells and bone marrow-derived DCs, into the mouse renal subcapsular space [Figure-2A]. In 2-3 weeks, it was observed that the graft behaved as an artificial lymph node (aLN), that possessed structures similar to secondary lymphoid tissues, such as $T$ and B cell segregated clusters, FDC and FRC networks in B cell and T cell areas respectively, together with HEV-like structures. The aLNs induced a strong secondary immune response in vivo, and the high responsiveness was confirmed by the accumulation of 
memory and effector T cells as well as memory B cells in the aLNs ${ }^{70}$. When the aLNs were re-transplanted into SCID mice, and followed by immunization, a robust secondary immune response was induced ${ }^{63,70}$.

In terms of an in vitro trial to create the human LN environment, it was demonstrated that antigen-specific B cell clusters could be observed in a bioreactor - an ex vivo culture device - containing a porous matrix with antigen-primed DCs ${ }^{75}$. Recently, potentially useful cell lines of artificial antigen-presenting cells (AAPC) were established for humans ${ }^{76}$. These cells possess the capacity to stimulate ex vivo or in vivo production of cytotoxic T cells from naïve $\mathrm{T}_{\text {cells }}{ }^{76}$. aAPC have also been made by covalently coupling antigen peptide-MHC complex and the B7.1 co-stimulatory molecule to magnetic beads. In vivo treatment with the aAPC induced significant or complete reduction of tumors in mice ${ }^{77}$. Based on this data, the aAPC might be an effective tool for tumor immunotherapy. The newly established aAPC might be also applicable for generating aLT. To apply artificial lymphoid tissues to human diseases, an in vivo human model would first have to be established, using knowledge from previous mouse experiments. There has been a much progress in the establishment and analysis of humanized mice ${ }^{78-80}$. NOD/SCID/IL-2 receptor $\gamma$ chain ${ }^{\text {null }}$ mice reconstituted with human $\mathrm{CD} 34+$ hematopoietic stem cells develop a functional human hematopoietic and immune system. As a result, humanized mice are utilized as a human disease model. They can be experimentally manipulated and can be used to directly study infectious diseases, immunological disorders, as well as cancer therapy in humans ${ }^{81-84}$. This model system could be a prime candidate for generating human aLT. 


\section{Concluding remarks}

The idea of generating aLT, especially tissue that is functionally similar to SLO and TLO, is a novel strategy to trigger ectopic adaptive immune responses in vivo, as well as to study the functions of these tissues. Progress in this area might be applied to both locally and systemic treat severe infection, autoimmune diseases, tumors, for example. From this point of view, trials for construction of human aLT are at a preliminary stage, but could be imperative in the future.

\section{Acknowledgements}

We thank Peter Burrows for helpful comments on the manuscript. This work was supported by the Grant-in-Aid for Scientific Research on Priority Areas from MEXT, Japan (Grant NO. 19059015)

Figure legends

Fugure 1: An overview of SLO and TLO formation 
Left: During embryogenesis, SLO formation is triggered by the interaction between LT $\alpha 1 \beta 2$ on LTi cells and LT $\beta R$ on LTo cells. LT $\beta R$ ligation leads to the expression of the adhesion molecules such as VCAM-1, ICAM-1 and MadCAM-1, lymphoid chemokines such as CXCL13, CCL19 and CCL21, RANKL and IL-7. RANKL and IL-7 receptor signaling on the recruited LTi cells further induces LT $\alpha 1 \beta 2$ to form a positive feedback loop for lymphoid tissue formation. Induction of VEGF-C production by LTo cell promotes lymphangiogenesis. Thus, LT $\beta$ R signaling in LTo cells attracts and retains hematopoietic cells, and leads to vascularization through HEV and lymphatic vessels. Right: TLO arise during inflammation, infection and in some autoimmune diseases. There have been reported circumstantial evidences indicated that antigen stimulation and/or LT $\beta R$ signaling play an important role in TLO formation ${ }^{8,9,12}$. At ectopic sites such as the synovial membrane or pancreas, specific stromal cell types produce chemokines in response to antigen stimulation. These stromal cells resemble LTo cells at lymphoid tissue anlagen of SLO and induce TLO formation. Both SLO and TLO have well-organized structures that include segregated T and B cell areas, DCs in T cell area, FDC networks in B cell follicles and vascular networks (lymphatic vessels and HEV). Migration and proper positioning of lymphoid tissue-associated cells is controlled by chemokines that essential for SLO and TLO development and function. CCL19 and CCL21 expressed by LTo and DCs attract and retain T cells within an appropriate area. On HEV, CCL21 facilitates migration of cells into lymphoid tissue. CXCL13 expressed by FDCs retains B cells in follicles.

Figure 2: Potential applications of artificial lymphoid tissue (aLT) 
Transplant of a collagen sponge containing bone marrow-derived and antigen-pulsed DCs and VCAM-1+ LT $\beta R+$ LTa overexpressing stromal cells into the renal subcapsular space produces the artificial lymphoid tissue (aLT) three weeks later. The tissue grafts resembles $L N$ and contains $T$ cell and $B$ cell clusters, FDC and FRC networks in B cell follicles and T cell areas respectively and HEV-like vascular structures ${ }^{63}$. The aLT contains memory B and T cells, follicular helper T cells (Tfh) and other helper T cell subsets. The presence of these cell types was confirmed by re-transplanting the aLT into SCID mice and followed by immunization, which gave a robust secondary immune response $[62,69]$. In these mice antigen-specific high-affinity IgG-producing $B$ cells were present in the spleen, LN and BM. The antigen-specific IgG reached 3-7 $\mathrm{mg} / \mathrm{ml}$ in the SCID mouse serum. Thus the aLT might be a suitable tool for the analysis of memory B cells and their niche, for characterization of Th and as an application to generate hybridomas secreting monoclonal high-affinity IgG class antibody with a high efficiency.

(B) aLT suppresses tumor growth in mice that have received tumor resection. When re-transplanted into SCID mice carrying the same tumors as in the original mice, aLT also suppress existing tumors. These data suggest that aLT may be applicable not only for enhancing antibody-mediated immune responses but also as a new strategy for cancer immunotherapy. In case of aLT use for immunotherapy, there might be a possibility that pathological lymphoid or myeloid cells co-opt the artificial lymphoid tissues and use the tissues to exacerbate the pathological response or induce unfavorable immune response(s) against the recipient. This point should be taken in consideration in a further trial for synthesis of artificial immune tissues/organs.

(C) aLT synthesized in the bacterial or viral antigen-immunized mice may also exhibit an 
ability to prevent a further spreading of the infection.

\section{References}

1 Mebius, R. (2003) Organogenesis of lymphoid tissues. Nat Rev Immunol 3 (4), 292-303

$2 \quad \mathrm{Fu}$, Y. and Chaplin, D. (1999) Development and maturation of secondary lymphoid tissues. Annu Rev Immunol 17, 399-433

3 Aloisi, F. and Pujol-Borrell, R. (2006) Lymphoid neogenesis in chronic inflammatory diseases. Nat Rev Immunol 6 (3), 205-217

4 Thaunat, O. et al. (2005) Lymphoid neogenesis in chronic rejection: evidence for a local humoral alloimmune response. Proc Natl Acad Sci US A 102 (41), 14723-14728

5 Young, C.L. et al. (1984) Immunohistologic characterization of synovial membrane lymphocytes in rheumatoid arthritis. Arthritis Rheum 27 (1), 32-39

6 Takemura, S. et al. (2001) Lymphoid neogenesis in rheumatoid synovitis. J Immunol 167 (2), 1072-1080

$7 \quad$ Shi, K. et al. (2001) Lymphoid chemokine B cell-attracting chemokine-1 (CXCL13) is expressed in germinal center of ectopic lymphoid follicles within the synovium of chronic arthritis patients. J Immunol 166 (1), 650-655

8 Grabner, R. et al. (2009) Lymphotoxin beta receptor signaling promotes tertiary lymphoid organogenesis in the aorta adventitia of aged ApoE-/- mice. $J$ Exp Med 206 (1), 233-248

9 Lotzer, K. et al. (2010) Mouse aorta smooth muscle cells differentiate into lymphoid tissue organizer-like cells on combined tumor necrosis factor receptor-1/lymphotoxin beta-receptor NF-kappaB signaling. Arterioscler Thromb Vasc Biol 30 (3), 395-402

10 Stott, D.I. et al. (1998) Antigen-driven clonal proliferation of B cells within the target tissue of an autoimmune disease. The salivary glands of patients with Sjogren's syndrome. J Clin Invest 102 (5), 938-946

11 Salomonsson, S. et al. (2003) Cellular basis of ectopic germinal center formation and autoantibody production in the target organ of patients with Sjogren's syndrome. Arthritis Rheum 48 (11), 3187-3201

12 Nasr, I.W. et al. (2007) Tertiary lymphoid tissues generate effector and memory T cells that lead to allograft rejection. Am J Transplant 7 (5), 1071-1079

13 Drayton, D.L. et al. (2006) Lymphoid organ development: from ontogeny to 
neogenesis. Nat Immunol 7 (4), 344-353

14 Ruddle, N.H. and Akirav, E.M. (2009) Secondary lymphoid organs: responding to genetic and environmental cues in ontogeny and the immune response. $J$ Immunol 183 (4), 2205-2212

15 Randall, T.D. et al. (2008) Development of secondary lymphoid organs. Annu Rev Immunol 26, 627-650

16 Kim, M.Y. et al. (2007) Function of CD4+CD3- cells in relation to B- and T-zone stroma in spleen. Blood 109 (4), 1602-1610

17 Kim, M.Y. et al. (2009) Lymphoid tissue inducer cells: architects of CD4 immune responses in mice and men. Clin Exp Immunol 157 (1), 20-26

18 Evans, I. and Kim, M.Y. (2009) Involvement of lymphoid inducer cells in the development of secondary and tertiary lymphoid structure. BMB Rep 42 (4), 189-193

19 Sun, Z. et al. (2000) Requirement for RORgamma in thymocyte survival and lymphoid organ development. Science 288 (5475), 2369-2373

20 Eberl, G. et al. (2004) An essential function for the nuclear receptor RORgamma(t) in the generation of fetal lymphoid tissue inducer cells. Nat Immunol 5 (1), 64-73

21 Kim, D. et al. (2000) Regulation of peripheral lymph node genesis by the tumor necrosis factor family member TRANCE. J Exp Med 192 (10), 1467-1478

22 Yokota, Y. et al. (1999) Development of peripheral lymphoid organs and natural killer cells depends on the helix-loop-helix inhibitor Id2. Nature 397 (6721), 702-706

23 Glanville, S.H. et al. (2009) Transplantation of embryonic spleen tissue reveals a role for adult non-lymphoid cells in initiating lymphoid tissue organization. Eur $J$ Immunol 39 (1), 280-289

24 Cupedo, T. et al. (2009) Human fetal lymphoid tissue-inducer cells are interleukin 17-producing precursors to RORC+ CD127+ natural killer-like cells. Nat Immunol $10(1), 66-74$

25 Buettner, M. et al. (2010) Stromal cell heterogeneity in lymphoid organs. Trends Immunol 31 (2), 80-86

26 Chyou, S. et al. (2008) Fibroblast-type reticular stromal cells regulate the lymph node vasculature. J Immunol 181 (6), 3887-3896

27 Molenaar, R. et al. (2009) Lymph node stromal cells support dendritic cell-induced gut-homing of T cells. J Immunol 183 (10), 6395-6402

28 Hammerschmidt, S.I. et al. (2008) Stromal mesenteric lymph node cells are essential for the generation of gut-homing T cells in vivo. J Exp Med 205 (11), 2483-2490

29 Iwata, M. et al. (2004) Retinoic acid imprints gut-homing specificity on T cells. Immunity 21 (4), 527-538 
Tomei, A.A. et al. (2009) Fluid flow regulates stromal cell organization and CCL21 expression in a tissue-engineered lymph node microenvironment. J Immunol 183 (7), 4273-4283

31 Peduto, L. et al. (2009) Inflammation recapitulates the ontogeny of lymphoid stromal cells. J Immunol 182 (9), 5789-5799

$32 \quad$ Fletcher, A.L. et al. (2010) Lymph node fibroblastic reticular cells directly present peripheral tissue antigen under steady-state and inflammatory conditions. $J$ Exp Med 207 (4), 689-697

33 Cohen, J.N. et al. (2010) Lymph node-resident lymphatic endothelial cells mediate peripheral tolerance via Aire-independent direct antigen presentation. $J$ Exp Med 207 (4), 681-688

34 Dejardin, E. et al. (2002) The lymphotoxin-beta receptor induces different patterns of gene expression via two NF-kappaB pathways. Immunity 17 (4), 525-535

35 Vondenhoff, M.F. et al. (2009) LTbetaR signaling induces cytokine expression and up-regulates lymphangiogenic factors in lymph node anlagen. J Immunol 182 (9), 5439-5445

36 Bista, P. et al. (2010) TRAF3 controls activation of the canonical and alternative NFkappaB by the lymphotoxin beta receptor. J Biol Chem 285 (17), 12971-12978

37 Xie, P. et al. (2007) Tumor necrosis factor receptor-associated factor 3 is a critical regulator of B cell homeostasis in secondary lymphoid organs. Immunity 27 (2), 253-267

38 GeurtsvanKessel, C.H. et al. (2009) Dendritic cells are crucial for maintenance of tertiary lymphoid structures in the lung of influenza virus-infected mice. $J$ Exp Med 206 (11), 2339-2349

39 Reijmers, R.M. et al. (2010) Impaired lymphoid organ development in mice lacking the heparan sulfate modifying enzyme glucuronyl C5-epimerase. J Immunol 184 (7), 3656-3664

40 Rodgers, K.D. et al. (2008) Heparan sulfate proteoglycans: a GAGgle of skeletal-hematopoietic regulators. Dev Dyn 237 (10), 2622-2642

41 Johnson, Z. et al. (2005) Interaction of chemokines and glycosaminoglycans: a new twist in the regulation of chemokine function with opportunities for therapeutic intervention. Cytokine Growth Factor Rev 16 (6), 625-636

42 Fukuyama, S. et al. (2006) Cutting edge: Uniqueness of lymphoid chemokine requirement for the initiation and maturation of nasopharynx-associated lymphoid tissue organogenesis. J Immunol 177 (7), 4276-4280

43 Bai, Z. et al. (2009) CXC chemokine ligand 12 promotes CCR7-dependent naive T 
cell trafficking to lymph nodes and Peyer's patches. J Immunol 182 (3), 1287-1295

44 Manzo, A. et al. (2007) CCL21 expression pattern of human secondary lymphoid organ stroma is conserved in inflammatory lesions with lymphoid neogenesis. $A m J$ Pathol 171 (5), 1549-1562

van de Pavert, S.A. et al. (2009) Chemokine CXCL13 is essential for lymph node initiation and is induced by retinoic acid and neuronal stimulation. Nat Immunol 10 (11), 1193-1199

46 Ansel, K.M. et al. (2000) A chemokine-driven positive feedback loop organizes lymphoid follicles. Nature 406 (6793), 309-314

47 Luther, S.A. et al. (2003) Overlapping roles of CXCL13, interleukin 7 receptor alpha, and CCR7 ligands in lymph node development. J Exp Med 197 (9), 1191-1198

48 Reif, K. et al. (2002) Balanced responsiveness to chemoattractants from adjacent zones determines B-cell position. Nature 416 (6876), 94-99

49 Fan, L. et al. (2000) Cutting edge: ectopic expression of the chemokine TCA4/SLC is sufficient to trigger lymphoid neogenesis. J Immunol 164 (8), 3955-3959

50 Luther, S.A. et al. (2002) Differing activities of homeostatic chemokines CCL19, CCL21, and CXCL12 in lymphocyte and dendritic cell recruitment and lymphoid neogenesis. J Immunol 169 (1), 424-433

51 Hjelmstrom, P. et al. (2000) Lymphoid tissue homing chemokines are expressed in chronic inflammation. Am J Pathol 156 (4), 1133-1138

52 Meier, D. et al. (2007) Ectopic lymphoid-organ development occurs through interleukin 7-mediated enhanced survival of lymphoid-tissue-inducer cells. Immunity 26 (5), 643-654

53 Drayton, D.L. et al. (2003) Ectopic LT alpha beta directs lymphoid organ neogenesis with concomitant expression of peripheral node addressin and a HEV-restricted sulfotransferase. J Exp Med 197 (9), 1153-1163

54 Akita, M. et al. (1997) Morphology of capillary-like structures in a three-dimensional aorta/collagen gel culture. Ann Anat 179 (2), 127-136

55 Niklason, L.E. et al. (1999) Functional arteries grown in vitro. Science 284 (5413), 489-493

$56 \mathrm{Yu}, \mathrm{X}$. et al. (2004) Bioreactor-based bone tissue engineering: the influence of dynamic flow on osteoblast phenotypic expression and matrix mineralization. Proc Natl Acad Sci U SA 101 (31), 11203-11208

57 Lutolf, M.P. et al. (2003) Repair of bone defects using synthetic mimetics of collagenous extracellular matrices. Nat Biotechnol 21 (5), 513-518

58 Teng, Y.D. et al. (2002) Functional recovery following traumatic spinal cord injury 
mediated by a unique polymer scaffold seeded with neural stem cells. Proc Natl Acad Sci USA 99 (5), 3024-3029

59 Yu, X. and Bellamkonda, R.V. (2003) Tissue-engineered scaffolds are effective alternatives to autografts for bridging peripheral nerve gaps. Tissue Eng 9 (3), $421-430$

60 Lutolf, M.P. and Hubbell, J.A. (2005) Synthetic biomaterials as instructive extracellular microenvironments for morphogenesis in tissue engineering. Nat Biotechnol 23 (1), 47-55

61 Griffith, L.G. and Swartz, M.A. (2006) Capturing complex 3D tissue physiology in vitro. Nat Rev Mol Cell Biol 7 (3), 211-224

62 Stachowiak, A.N. and Irvine, D.J. (2008) Inverse opal hydrogel-collagen composite scaffolds as a supportive microenvironment for immune cell migration. $J$ Biomed Mater Res A 85 (3), 815-828

63 Suematsu, S. and Watanabe, T. (2004) Generation of a synthetic lymphoid tissue-like organoid in mice. Nat Biotechnol 22 (12), 1539-1545

64 Richardson, T.P. et al. (2001) Polymeric system for dual growth factor delivery. Nat Biotechnol 19 (11), 1029-1034

65 Sun, Q. et al. (2005) Sustained vascular endothelial growth factor delivery enhances angiogenesis and perfusion in ischemic hind limb. Pharm Res 22 (7), 1110-1116

66 Chen, R.R. et al. (2007) Spatio-temporal VEGF and PDGF delivery patterns blood vessel formation and maturation. Pharm Res 24 (2), 258-264

67 Thompson, E.D. et al. Tumor masses support naive T cell infiltration, activation, and differentiation into effectors. The Journal of Experimental Medicine

68 Zou, W. (2005) Immunosuppressive networks in the tumour environment and their therapeutic relevance. Nat Rev Cancer 5 (4), 263-274

69 Flavell, R.A. et al. (2010) The polarization of immune cells in the tumour environment by TGFbeta. Nat Rev Immunol 10 (8), 554-567

70 Okamoto, N. et al. (2007) Artificial lymph nodes induce potent secondary immune responses in naive and immunodeficient mice. J Clin Invest 117 (4), 997-1007

71 Pan, W.R. et al. (2008) Senile changes in human lymph nodes. Lymphat Res Biol 6 (2), $77-83$

72 Hadamitzky, C. et al. (2010) Age-dependent histoarchitectural changes in human lymph nodes: an underestimated process with clinical relevance? J Anat 216 (5), 556-562

73 Willfuhr, K.U. et al. (1992) Splenic autotransplantation provides protection against fatal sepsis in young but not in old rats. J Pediatr Surg 27 (9), 1207-1212 
74 Grikscheit, T.C. et al. (2008) Tissue-engineered spleen protects against overwhelming pneumococcal sepsis in a rodent model. J Surg Res 149 (2), 214-218

75 Giese, C. et al. (2006) A human lymph node in vitro--challenges and progress. Artif Organs 30 (10), 803-808

76 Butler, M.O. et al. (2007) Long-lived antitumor CD8+ lymphocytes for adoptive therapy generated using an artificial antigen-presenting cell. Clin Cancer Res 13 (6), 1857-1867

77 Ugel, S. et al. (2009) In vivo administration of artificial antigen-presenting cells activates low-avidity T cells for treatment of cancer. Cancer Res 69 (24), 9376-9384

78 Ito, M. et al. (2002) NOD/SCID/gamma(c)(null) mouse: an excellent recipient mouse model for engraftment of human cells. Blood 100 (9), 3175-3182

79 Shultz, L.D. et al. (2005) Human lymphoid and myeloid cell development in NOD/LtSz-scid IL2R gamma null mice engrafted with mobilized human hemopoietic stem cells. J Immunol 174 (10), 6477-6489

80 Ishikawa, F. et al. (2005) Development of functional human blood and immune systems in NOD/SCID/IL2 receptor \{gamma\} chain(null) mice. Blood 106 (5), 1565-1573

81 Shultz, L.D. et al. (2007) Humanized mice in translational biomedical research. Nat Rev Immunol 7 (2), 118-130

82 Saito, Y. et al. (2010) Induction of cell cycle entry eliminates human leukemia stem cells in a mouse model of AML. Nat Biotechnol 28 (3), 275-280

83 Zhang, L. et al. (2007) HIV-1 infection and pathogenesis in a novel humanized mouse model. Blood 109 (7), 2978-2981

84 Legrand, N. et al. (2009) Humanized mice for modeling human infectious disease: challenges, progress, and outlook. Cell Host Microbe 6 (1), 5-9 


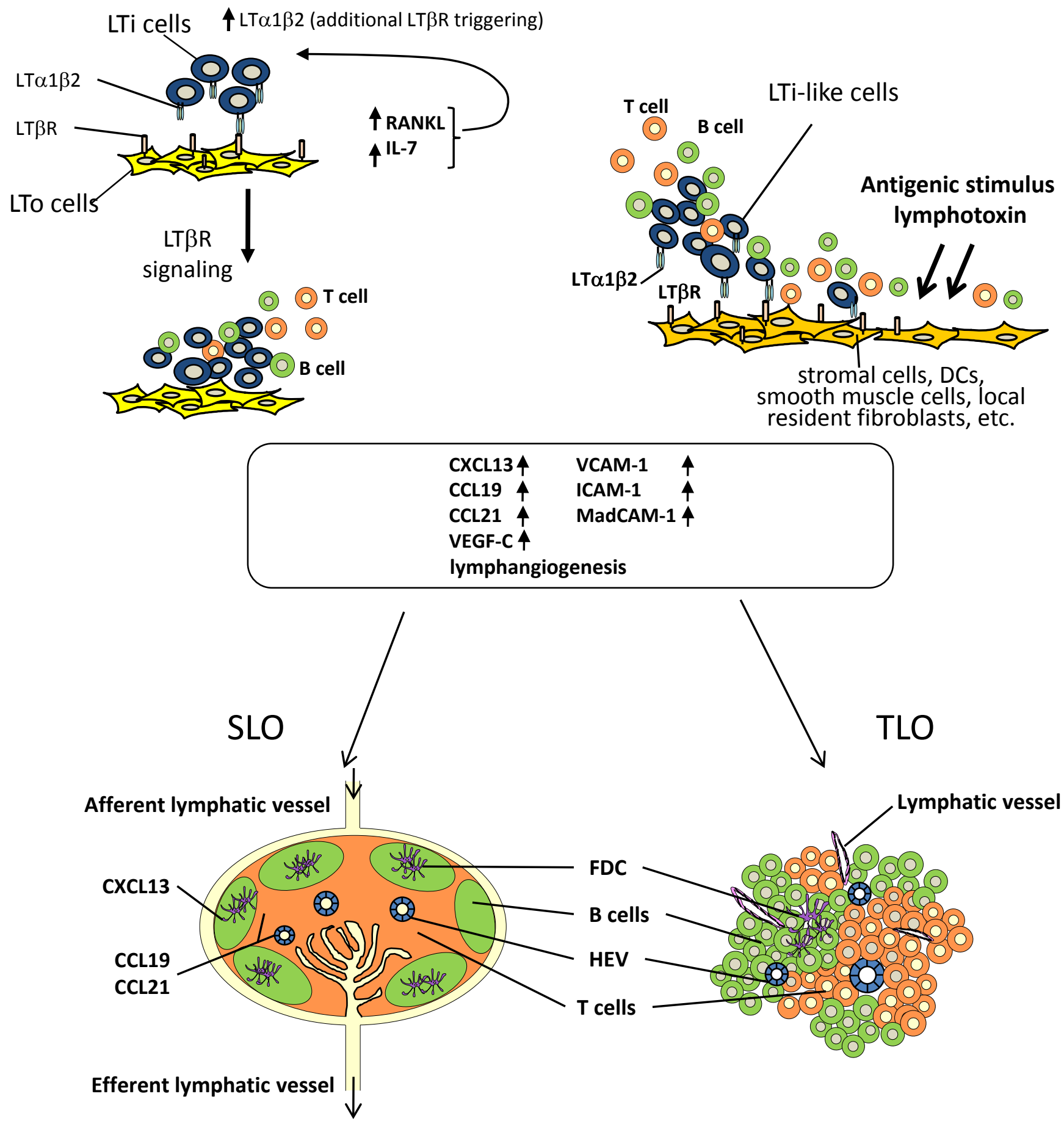


(A) Collagen sponge (containing

(A) stromal cell and antigen-pulsed
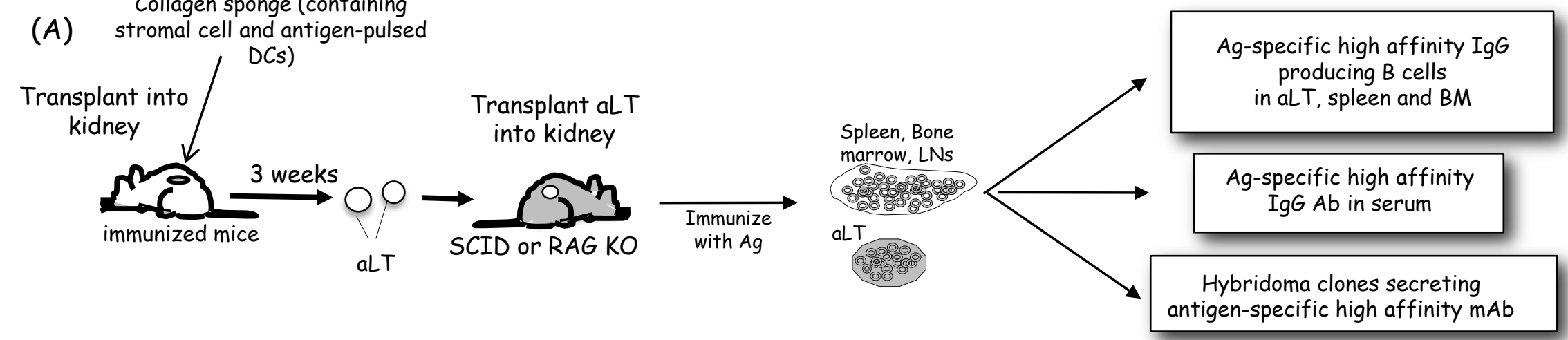

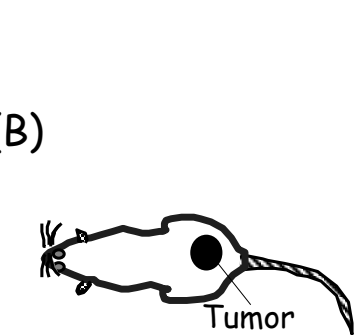

Tumor-bearing mouse with Ag
Collagen sponges

(stromal cell, antigen-pulsed DCs) kidney
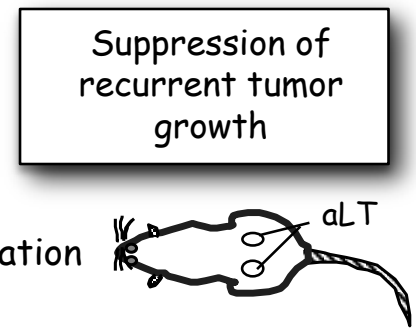

aLT formation

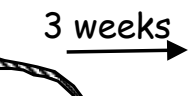

Resection

of original tumors

$>$

No aLT formation
Without transplant

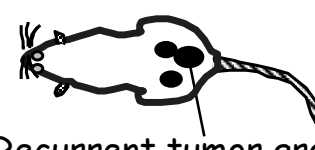

Recurrent tumor growth

\section{Transplant aLT into} tumor-bearing SCID mice aLT Tumor
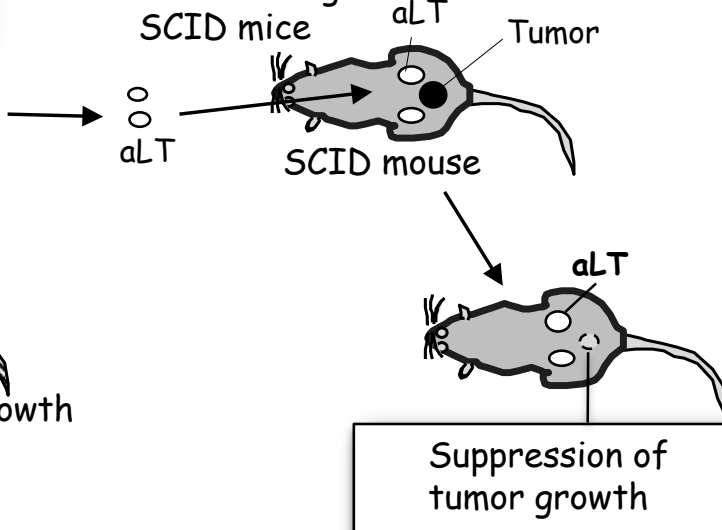

Transplant aLT into renal subcapsular space

(C)

Collagen sponges
(stromal cell, DCs

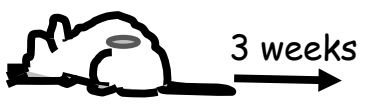

Immunized with bacterial or viral antigen

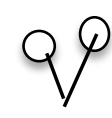

aLT

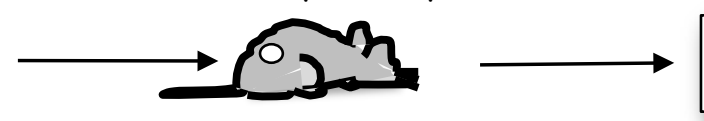

SCID mice with bacterial or viral infection 